\title{
RESURGENCE OF THE FRACTIONAL POLYLOGARITHMS
}

\author{
Ovidiu Costin and Stavros Garoufalidis
}

\begin{abstract}
The fractional polylogarithms, depending on a complex parameter $\alpha$, are defined by a series which is analytic inside the unit disk. After an elementary conversion of the series into an integral presentation, we show that the fractional polylogarithms are multivalued analytic functions in the complex plane minus 0 and 1 . For non-integer values of $\alpha$, we prove the analytic continuation, compute the monodromy around 0 and 1, give a Mittag-Leffler decomposition and compute the asymptotic behavior for large values of the complex variable. The fractional polylogarithms are building blocks of resurgent functions that are used in proving that certain power series associated with knotted objects are resurgent. This is explained in a separate publication [CG3]. The motivic or physical interpretation of the monodromy of the fractional polylogarithms for non-integer values of $\alpha$ is unknown to the authors.
\end{abstract}

\section{Introduction}

1.1. The fractional polylogarithm and its history. For a complex number $\alpha$, let us define the $\alpha$-polylogarithm function $\operatorname{Li}_{\alpha}(z)$ by the following series:

$$
\operatorname{Li}_{\alpha}(z)=\sum_{n=1}^{\infty} \frac{z^{n}}{n^{\alpha}}
$$

which is absolutely convergent for $|z|<1$. These functions appear in algebraic geometry, number theory, mathematical physics, applied mathematics and the theory of special functions. Since

$$
z \frac{d}{d z} \operatorname{Li}_{\alpha}(z)=\operatorname{Li}_{\alpha-1}(z)
$$

we really need to study $\operatorname{Li}_{\alpha}(z)$ for $\alpha \bmod \mathbb{Z}$.

For integer $\alpha$ a lot is known about the $\alpha$-polylogarithm. For example, $\operatorname{Li}_{0}(z)=$ $1 /(1-z)$, thus (2) implies that for all $\alpha \in \mathbb{Z}^{-}, \operatorname{Li}_{\alpha}(z) \in \mathbb{Q}(z)$ is a rational function with a single singularity at $z=1$.

When $\alpha \in \mathbb{N}$, the functions $\operatorname{Li}_{\alpha}(z)$ were studied in the nineteenth century, forgotten for many years, and rediscovered by the algebraic geometers in the late 1970s; see for example Lewin's book [Lw], Bloch's paper [Bl] and the survey articles [Oe, Za1, Za2].

Received by the editors February 9, 2008

1991 Mathematics Subject Classification. Primary 57N10. Secondary 57M25.

Key words and phrases. fractional polylogarithms, resurgence, Écalle, Mittag-Leffler decomposition, monodromy, motives, asymptotics, Appell's equation, Lambert function.

O.C. was supported in part by NSF grants DMS-0406193 and DMS-0600369 and S.G was supported in part by NSF grant DMS-0505445. 
It is well known that $\operatorname{Li}_{\alpha}(z)$ is a multivalued function defined on $\mathbb{C} \backslash\{0,1\}$ with computable monodromy; see [We, Ha, BD, Oe] and [MPV]. For $\alpha \in \mathbb{N}$, the $\alpha$ polylogarithms are special functions that play a key role in algebraic geometry. For $\alpha \in \mathbb{N}$, the special values

$$
\operatorname{Li}_{\alpha}(1)=\zeta(\alpha)
$$

are well-known examples of periods; see [KZ]. This is not an accident. Zagier and Deligne conjectured that special values (at integers) of $L$-functions of algebraic varieties are expressed by the $\alpha$-polylogarithm for $\alpha \in \mathbb{N}$; see [Za1] and [De]. A motivic interpretation of $\operatorname{Li}_{\alpha}(z)$ for $\alpha \in \mathbb{N}$ is given in [BD], as well as a conjecture that the $\alpha$-th Beilinson-Deligne regulator maps are expressed by the $\alpha$-polylogarithm for $\alpha \in \mathbb{N}$.

For integer $\alpha$, elliptic polylogarithms that resemble $\operatorname{Li}_{\alpha}\left(e^{z}\right)$ were introduced by Beilinson-Levin in [BL], and further studied in $[\mathrm{Lv}]$ in relation to motivic cohomology conjectures for elliptic curves. For a recent survey on the better-known dilogarithm, see $[\mathrm{Za} 2]$.

The $\alpha$-polylogarithms for noninteger $\alpha$ are also classical and modern objects. They were studied in the eighteenth century by Jonquière as a function of two complex variables $\alpha$ and $z$; see [Jo]. Several approximation formulas were obtained by Jonquière and half a century later by Truesdell, whose motivation was asymptotic properties of polymer structures; see [Tr]. Further results regarding approximation and analytic continuation were obtained by Pickard in the sixties, and more recently by KenyonWilson in relation to resonance of some statistical mechanical models; see [Pi, KW] and also [CLZ, Prop.1].

The $\alpha$-polylogarithm functions for half-integer $\alpha$ appear naturally in the context of an Euler-MacLaurin summation, and are also used in proving resurgence of some power series associated to knotted objects; see [CG2] and [CG3]. They also play a prominent role in proving analytic continuation of some power series that encode quantum invariants of knotted objects; see for example [Ga, Sec.7].

In addition, in 1994, M. Kruskal proposed to the first author to study the analytic continuation and the global bahavior of the function $\mathrm{Li}_{1 / 2}(z)$. This problem was a motivation for a global reconstruction theorem of resurgent functions from local data, developed by the first author several years ago (and independently by Écalle in [Ec2]), and recently written in $[\mathrm{C}]$.

The purpose of this short note is to study the

(a) the analytic continuation

(b) the Mittag-Leffler decomposition

(c) the asymptotic behavior for large $|z|$

of the polylogarithm function $\operatorname{Li}_{\alpha}(z)$ for non-integer $\alpha$. With over a century of history on the fractional polylogarithm, some of our results resemble statements of the above mentioned literature. However, we were not able to find the key Equation (13), nor an explicit computation of the monodromy around $z=0$ and $z=1$ in the literature. The latter does not seem to have a finite dimensional faithful representation, and its motivic or physical origin is unknown when $\alpha \in \mathbb{Q} \backslash \mathbb{Z}$.

1.2. Statement of the results. Let us recall first what is a multivalued analytic function on $\mathbb{C} \backslash\{0,1\}$. Such functions are examples of global analytic functions (see 
[Ah]) and examples of resurgent functions in the sense of Écalle, [Ec1]. Let $X$ denote the universal cover of $(\mathbb{C} \backslash\{0,1\}, 1 / 2)$ with base point at $1 / 2$. As a set, we have:

$$
X=\left\{\text { homotopy classes }[c] \text { of paths } c \text { in } \mathbb{C} \backslash\{0,1\} \text { starting at } \frac{1}{2}\right\} .
$$

There is an action of $F=\pi_{1}(\mathbb{C} \backslash\{0,1\}, 1 / 2)$ on $X$ given by $g \cdot[c]=[\gamma . c]$ for $g=[\alpha \gamma]$ and $[c] \in X$. By a multivalued analytic (in short, resurgent) function $f$ on $\mathbb{C} \backslash\{0,1\}$ we mean an analytic function on $X$. For $[c] \in X$, where $c$ is a path from $1 / 2$ to $z$, we write, following [Oe]:

$$
f^{[c]}(z):=f([c]) .
$$

Observe that $F$ is a free group on $\left[c_{0}\right]$ and $\left[c_{1}\right]$, where

$$
c_{0}(t)=\frac{1}{2} e^{2 \pi i t}, \quad c_{1}(t)=1+\frac{1}{2} e^{2 \pi i t}
$$

are paths around 0 and 1 respectively:

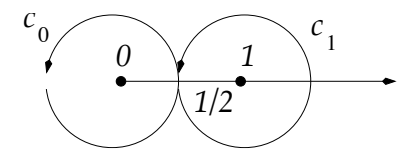

In what follows, $\alpha$ is not an integer. Let us introduce some useful notation. Let $\gamma$ denote a Hankel contour that encircles the positive real axis: The next definition

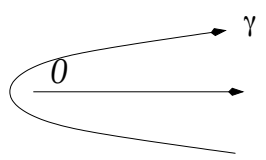

uses notation familiar to algebraic geometry. See for example the survey paper [Oe].

Definition 1.1. For $\alpha \in \mathbb{C} \backslash \mathbb{Z}$, let $M_{\alpha}(z)$ denote the multivalued function on $\mathbb{C} \backslash\{0,1\}$ given by:

$$
M_{\alpha}(z)=C_{\alpha}(\log z)^{\alpha-1}
$$

where

$$
C_{\alpha}=e^{\pi i(-\alpha-1)} \Gamma(1-\alpha)
$$

For $k \in \mathbb{Z}$, let us define the twisted multivalued functions $M_{\alpha}[k](z)$ for $z \in \mathbb{C} \backslash\{0,1\}$ by:

$$
M_{\alpha}[k](z):=M_{\alpha}\left(z e^{2 \pi i k}\right)=C_{\alpha}(\log z+2 \pi i k)^{\alpha-1} .
$$

The following theorem converts the series $(1)$ of $\operatorname{Li}_{\alpha}(z)$ into an integral, from which we can easily deduce the existence of analytic continuation.

Theorem 1. (a) For $|z|<1$ and $\alpha$ such that $\operatorname{Re}(\alpha)>0, \operatorname{Li}_{\alpha}(z)$ has an integral representation:

$$
\operatorname{Li}_{\alpha}(z)=\frac{1}{\Gamma(\alpha)} \int_{0}^{\infty} q^{\alpha-1} \frac{z}{e^{q}-z} d q
$$


known as Appell's integral in [Tr, Sec.2].

(b) For $|z|<1$ and $\alpha \in \mathbb{C} \backslash \mathbb{Z}, \operatorname{Li}_{\alpha}(z)$ has an integral representation:

$$
\operatorname{Li}_{\alpha}(z)=\frac{C_{\alpha}}{2 \pi i} \int_{\gamma} q^{\alpha-1} \frac{z}{e^{q}-z} d q
$$

(c) For all $\alpha \in \mathbb{C} \backslash \mathbb{Z}, \operatorname{Li}_{\alpha}(z)$ has an analytic continuation to a multivalued function on $\mathbb{C} \backslash\{0,1\}$. More precisely, let $z \in \mathbb{C} \backslash\{0,1\}$ and $c$ any path from $1 / 2$ to $z$ in $\mathbb{C} \backslash\{0,1\}$. Then, we have:

$$
\begin{array}{ll}
\mathrm{Li}_{\alpha}^{\left[c_{0} c\right]}(z)=\mathrm{Li}_{\alpha}^{[c]}(z) & \operatorname{Li}_{\alpha}^{\left[c_{1} c\right]}(z)=\mathrm{Li}_{\alpha}^{[c]}(z)-\left(1-e^{2 \pi i \alpha}\right) M_{\alpha}^{[c]}(z) \\
M_{\alpha}^{\left[c_{0} c\right]}(z)=M_{\alpha}^{[c]}[1](z) & M_{\alpha}^{\left[c_{1} c\right]}(z)=-\left(1-e^{2 \pi i \alpha}\right) M_{\alpha}^{[c]}(z)
\end{array}
$$

(d) For $\alpha$ such that $\operatorname{Re}(\alpha)<0, \operatorname{Li}_{\alpha}(z)$ has a Mittag-Leffler type decomposition:

$$
\operatorname{Li}_{\alpha}(z)=C_{\alpha}\left((\log z)^{\alpha-1}+\sum_{k=1}^{\infty}(\log z+2 \pi i k)^{\alpha-1}+(\log z-2 \pi i k)^{\alpha-1}\right)
$$

where the series is uniformly convergent on compact sets. Thus, we have:

$$
\mathrm{Li}_{\alpha}=\sum_{k \in \mathbb{Z}} M_{\alpha}[k]:=\lim _{N \rightarrow \infty} \sum_{k=-N}^{N} M_{\alpha}[k] .
$$

When $\alpha$ is a negative integer, the right hand side of (14) is an Eisenstein series; see [Ap]. The Mittag-Leffler decomposition (14) is an analogue of Hurwitz's theorem; see $[\mathrm{Ap}]$. The Mittag-Leffler (14) implies is the following corollary.

Corollary 1.2. For $\alpha$ such that $\operatorname{Re}(\alpha)<0$ and $z$ such that $\operatorname{Re}(z)<0$ and $|z|<2 \pi$ we have:

$$
\mathrm{Li}_{\alpha}\left(e^{z}\right)=C_{\alpha} z^{\alpha-1}+\sum_{n=0}^{\infty} \frac{\zeta(\alpha-n)}{n !} z^{n}
$$

Compare with [CLZ, Prop.1].

The integral formula (9) and some stationary phase implies the following estimate for the behavior of the fractional polylogarithms for large $|z|$.

Corollary 1.3. For $\operatorname{Re}(\alpha)>0$ and $z$ large we have:

$$
\operatorname{Li}_{\alpha}(z)=-\frac{1}{\Gamma(\alpha+1)}\left((\log z)^{\alpha}+o(1)\right) .
$$

For $\alpha \in \mathbb{N}$, this is known; see [Oe, Eqn.7].

1.3. Plan of the proof. Once we convert the series definition of the $\alpha$-polylogarithms into an integral formula, analytic continuation follows from a general principle, i.e., by moving the contour of integration and achieving analytic continuation. If we move the contour of integration to $-\infty$, and the integral vanishes at $-\infty$, collecting residues gives a Mittag-Leffler type decomposition of $\operatorname{Li}_{\alpha}(z)$ for $\alpha<0$, $\alpha \notin \mathbb{Z}$. 
1.4. Acknowledgement. An early version of this paper was presented at talks in Orsay and the University of Maryland in the fall of 2006. The authors wish to thank J. Écalle for encouraging conversations. M. Kontsevich pointed out to the second author that some aspects of the fractional polylogarithms have been studied independently by M. Kontsevich and D. Zagier. After the paper was written, J. Morava informed us of $[\mathrm{EM}]$, where the fractional polylogarithms are also studied from the point of view of distributions over the real numbers.

\section{Proofs}

2.1. Proof of Theorem 1. In this section we give a proof of Theorem 1 .

Proof. (a) For $\operatorname{Re}(\alpha)>0$ and $n \in \mathbb{N}^{+}$we have:

$$
\frac{1}{n^{\alpha}}=\frac{1}{\Gamma(\alpha)} \int_{0}^{\infty} q^{\alpha-1} e^{-n q} d q
$$

Interchanging summation and integration (valid for $|z|<1$ ) gives:

$$
\begin{aligned}
\operatorname{Li}_{\alpha}(z) & =\sum_{n=1}^{\infty} \frac{1}{n^{\alpha}} z^{n} \\
& =\frac{1}{\Gamma(\alpha)} \int_{0}^{\infty} q^{\alpha-1} \sum_{n=1}^{\infty}\left(z e^{-q}\right)^{n} d q \\
& =\frac{1}{\Gamma(\alpha)} \int_{0}^{\infty} q^{\alpha-1} \frac{z}{e^{q}-z} d q
\end{aligned}
$$

(b) Let

$$
I_{\alpha}(z)=C_{\alpha} \int_{\gamma} q^{\alpha-1} \frac{z}{e^{q}-z} d q
$$

denote the right hand side of Equation (10). Observe that $I_{\alpha}(z)$ is well-defined for $\alpha \in \mathbb{C} \backslash \mathbb{Z}$, and $z \in \mathbb{C} \backslash[1, \infty)$.

Since for fixed $z$ inside the unit disk, both sides of (10) are analytic functions of $\alpha \in \mathbb{C} \backslash \mathbb{Z}$, it suffices to prove (10) for $\operatorname{Re}(\alpha)>0, \alpha \notin \mathbb{Z}$. We claim that for such $\alpha$, we have:

$$
\int_{0}^{\infty} q^{\alpha-1} \frac{z}{e^{q}-z} d q=\frac{1}{1-e^{2 \pi i \alpha}} \int_{\gamma} q^{\alpha-1} \frac{z}{e^{q}-z} d q
$$

Indeed, we push the Hankel contour $\gamma$ until its upper (resp. lower) part touches $\mathbb{R}^{+}$ from above (resp. below) and push the tip of the contour to touch zero. On the upper part we have $q^{\alpha-1}=|q|^{\alpha-1}$, and on the lower side we have

$$
q^{\alpha-1}=e^{(\alpha-1) \ln |q|+2 \pi i(\alpha-1)}=|q|^{\alpha-1} e^{2 \pi i \alpha} .
$$

Moreover, the upper integral is traversed in the direction $(0, \infty)$ while the lower one is traversed from $(\infty, 0)$. We thus get

$$
\int_{\gamma} q^{\alpha-1} \frac{1}{e^{q}-z} d q=\left(1-e^{2 \pi i \alpha}\right) \int_{0}^{\infty} q^{\alpha-1} \frac{1}{e^{q}-z} d q
$$


Thus, (18) follows. Since for $\alpha \in \mathbb{C} \backslash \mathbb{Z}$ the $\Gamma$ function satisfies the the reflexion symmetry (see eg. [Ol]):

$$
\frac{1}{\left(1-e^{2 \pi i \alpha}\right) \Gamma(\alpha)}=\frac{e^{\pi i(-\alpha-1)} \Gamma(1-\alpha)}{2 \pi i}=\frac{C_{\alpha}}{2 \pi i},
$$

(b) follows.

(c) Fix $\alpha$ such that $\operatorname{Re}(\alpha)>0, \alpha \notin \mathbb{Z}$. The integral representation (9) analytically continues $\operatorname{Li}_{\alpha}(z)$ for $z$ in the cut plane $\mathbb{C} \backslash[1, \infty)$. Let us compute the variation (i.e., jump) of the function across the cut $z \in(1, \infty)$. Changing variable to $e^{q}=x$ in (9), we have:

$$
\operatorname{Li}_{\alpha}(z)=\frac{1}{\Gamma(\alpha)} \int_{1}^{\infty} \frac{(\log x)^{\alpha-1}}{x} \frac{z}{x-z} d x
$$

Fix $z \in(1, \infty)$. Then the above equality gives by contour deformation and Cauchy's theorem (see eg. [Df]):

$$
\begin{aligned}
\lim _{\epsilon \rightarrow 0^{+}}\left(\operatorname{Li}_{\alpha}(z+i \epsilon)-\operatorname{Li}_{\alpha}(z-i \epsilon)\right) & =\frac{2 \pi i}{\Gamma(\alpha)} \operatorname{Res}\left(\frac{(\log x)^{\alpha-1}}{x} \frac{z}{x-z}, x=z\right) \\
& =\frac{2 \pi i}{\Gamma(\alpha)}(\log z)^{\alpha-1} \\
& =\left(1-e^{2 \pi i \alpha}\right) M_{\alpha}(z) .
\end{aligned}
$$

On the other hand, Equation (19) implies that

$$
\lim _{\epsilon \rightarrow 0^{+}}\left(M_{\alpha}(z+i \epsilon)-M_{\alpha}(z-i \epsilon)\right)=\left(1-e^{2 \pi i \alpha}\right) M_{\alpha}(z) .
$$

Since

$$
f^{\left[c_{1} c\right]}(z)=-\lim _{\epsilon \rightarrow 0^{+}}\left(f^{[c]}(z+i \epsilon)-f^{[c]}(z-i \epsilon)\right),
$$

the above equations imply that

$$
\begin{aligned}
\mathrm{Li}_{\alpha}^{\left[c_{1} c\right]}(z) & =\mathrm{Li}_{\alpha}^{[c]}(z)-\left(1-e^{2 \pi i \alpha}\right) M_{\alpha}^{[c]}(z) \\
M_{\alpha}^{\left[c_{1} c\right]}(z) & =-\left(1-e^{2 \pi i \alpha}\right) M_{\alpha}^{[c]}(z) .
\end{aligned}
$$

On the other hand, (9) defines an analytic function for $z \in(-\infty, 0)$, and the monodromy of $M_{\alpha}(z)$ for $z=0$ can be computed from the definition of $M_{\alpha}(z)$. Thus, we obtain

$$
\begin{aligned}
\mathrm{Li}_{\alpha}^{\left[c_{0} c\right]}(z) & =\mathrm{Li}_{\alpha}^{[c]}(z) \\
M_{\alpha}^{\left[c_{0} c\right]}(z) & =M_{\alpha}^{[c]}[1](z) .
\end{aligned}
$$

This proves that when $\operatorname{Re}(\alpha)>0, \alpha \notin \mathbb{Z}, \operatorname{Li}_{\alpha}(z)$ is a multivalued function on $\mathbb{C} \backslash\{0,1\}$ with monodromy given by (11) and (12). If $\alpha \in \mathbb{C} \backslash \mathbb{Z}$, use (2), the fact

$$
z \frac{d}{d z} M_{\alpha}(z)=M_{\alpha-1}(z)
$$

and differentiation to conclude (c).

(d) Since both sides of (13) are analytic functions of $\alpha$ for fixed $z$, it suffices to prove (13) for $\alpha$ such that $\operatorname{Re}(\alpha)<0, \alpha \notin \mathbb{Z}$. For such $\alpha$, we will use the integral representation of $\operatorname{Li}_{\alpha}(z)$ given by (10). Fix a complex number $z \in \mathbb{C} \backslash[1, \infty)$ and the Hankel contour $\gamma$ which separates the plane into two regions so that $2 \pi i k+\log z$ 
lies in the region that contains $-\infty$ for all $k \in \mathbb{Z}$. This is possible since the points $2 \pi i k+\log z \operatorname{lie}$ in a vertical line. Now, push the Hankel contour to the left, and deform it to $-\infty$. Since $\operatorname{Re}(\alpha)<0$, the integral vanishes when the contour is deformed to $-\infty$. In the process of deformation, we apply Cauchy's theorem and collect residues at the singularities. The singularities of the integrand are simple poles at the points where $q=\log z+2 \pi i k$ for integer $k$. The residue is given by:

$$
\operatorname{Res}\left(q^{\alpha-1} \frac{z}{e^{q}-z}, q=\log z+2 \pi i k\right)=(\log z+2 \pi i k)^{\alpha-1}
$$

When we push the contour to $-\infty$, we collect the series (13) which is absolutely convergent on compact sets. The result follows.

Remark 2.1. Part (b) of Theorem 1 states that for all $\alpha \in \mathbb{C} \backslash \mathbb{Z}$ and $|z|<1$ we have:

$$
\operatorname{Li}_{\alpha}(z)=I_{\alpha}(z) .
$$

Moreover, $\operatorname{Li}_{\alpha}(z)$ satisfies the differential equation (2). It is easy to show independently from Theorem 1 that for every $\alpha \in \mathbb{C} \backslash \mathbb{Z}, I_{\alpha}(z)$ satisfies the differential equation

$$
z \frac{d}{d z} I_{\alpha}(z)=I_{\alpha-1}(z)
$$

Indeed, use the algebraic identity:

$$
\frac{d}{d z} \frac{z}{e^{q}-z}=\frac{e^{q}}{\left(e^{q}-z\right)^{2}}=\frac{d}{d q} \frac{-1}{e^{q}-z}
$$

After differentiation and integration by parts, we have:

$$
\begin{aligned}
z I_{\alpha}^{\prime}(z) & =C_{\alpha} z \int_{\gamma} q^{\alpha-1} \frac{e^{q}}{\left(e^{q}-z\right)^{2}} d q \\
& =C_{\alpha} z \int_{\gamma} q^{\alpha-1} \frac{d}{d q} \frac{-1}{e^{q}-z} d q \\
& =C_{\alpha}(\alpha-1) z \int_{\gamma} q^{\alpha-2} \frac{1}{e^{q}-z} d q \\
& =C_{\alpha-1} z \int_{\gamma} q^{\alpha-2} \frac{1}{e^{q}-z} d q \\
& =I_{\alpha-1}(z) .
\end{aligned}
$$

Remark 2.2. An alternative way to prove part (d) of Theorem 1 is to use the MittagLeffler decomposition of the function $q \rightarrow 1 /\left(e^{q}-z\right)$ (see [Cn, Sec.V])

$$
\frac{z}{e^{q}-z}=-\frac{1}{2}+\frac{1}{q-\log z}+\sum_{k=1}^{\infty} \frac{1}{q-\log z+2 \pi i k}+\frac{1}{q-\log z-2 \pi i k},
$$

interchange summation and integration in (17) and use the fact that

$$
\int_{\gamma} q^{\alpha-1} \frac{d q}{q-\log z-2 \pi i k}=2 \pi i(\log z+2 \pi i k)^{\alpha-1}
$$


Remark 2.3. In Écalle's language, (18) is a special case of

$$
\int_{0}^{\infty} f(q) d q=\frac{1}{1-e^{2 \pi i \alpha}} \int_{\gamma} \check{f}(q) d q
$$

together with the fact that if $f(q)=q^{\alpha-1}$, then $\check{f}(q)=\left(1-e^{2 \pi i \alpha}\right)^{-1} f(q)$. For a self-contained introduction to majors/minors, see also [Ma].

Remark 2.4. The Mittag-Leffler type decomposition (13) implies that $\operatorname{Li}_{\alpha}(z)$ is multivalued on $\mathbb{C} \backslash\{0,1\}$, for all $\alpha$ such that $\operatorname{Re}(\alpha)<0, \alpha \notin \mathbb{Z}$. It also implies Equations (11) and (12). Indeed, for $z$ near 1 and $k \neq 0, M_{\alpha}(z)$ is analytic. Thus, (13) implies that for $z$ near 1 we have

$$
\operatorname{Li}_{\alpha}(z)=M_{\alpha}(z)+h(z)
$$

where $h$ is analytic for $z$ near 1 . This proves the second part of Equation (11). If $z$ is near 0 , then

$$
\begin{aligned}
\operatorname{Li}_{\alpha}\left(z e^{2 \pi i}\right)-\operatorname{Li}_{\alpha}(z)= & M_{\alpha}[1](z)+\sum_{k=1}^{\infty} M_{\alpha}[k+1](z)+M_{\alpha}[-k+1](z) \\
& -M_{\alpha}[0](z)-\sum_{k=1}^{\infty} M_{\alpha}[k](z)+M_{\alpha}[-k](z)=0 .
\end{aligned}
$$

This implies the first part of Equation (11). Equation (12) follows easily from the definition of $M_{\alpha}(z)$.

2.2. Proof of Corollaries 1.2 and 1.3. Corollary 1.2 follows by expanding the sum in (13) as a convergent power series in $\log z$, and using the functional equation for the Riemann zeta function:

$$
\zeta(s)=2^{s} \pi^{s-1} \sin \left(\frac{\pi s}{2}\right) \Gamma(1-s) \zeta(1-s) .
$$

Compare with [CLZ, Prop.1].

To prove Corollary 1.3, let us fix $\alpha$ with $\operatorname{Re}(\alpha)>0$ and consider the right hand side of Equation (9), which makes sense for $z \in \mathbb{C} \backslash[1, \infty)$. The idea is to make some changes of variables and integration by parts. Let us fix an angle $\theta \in(0,2 \pi)$ and consider a complex $z=|z| e^{i \theta}$ with $|z|$ large.

Making the change of variables $q=\log \tau$, integrating by parts, and making a change of variables $\tau=z+s$ and $s=z t$, we obtain that:

$$
\begin{aligned}
z \int_{0}^{\infty} q^{\alpha-1} \frac{z}{e^{q}-z} d q & =z \int_{1}^{\infty} \frac{(\log \tau)^{\alpha-1}}{\tau-z} \frac{d \tau}{\tau}=\frac{z}{\alpha} \int_{1}^{\infty} \frac{1}{\tau-z} d\left((\log \tau)^{\alpha}\right) \\
& =\frac{z}{\alpha} \int_{1}^{\infty} \frac{(\log \tau)^{\alpha}}{(\tau-z)^{2}} d \tau=\frac{z}{\alpha} \int_{1-z}^{\infty} \frac{(\log (z+s))^{\alpha}}{s^{2}} d s \\
& =\frac{1}{\alpha} \int_{1 / z-1}^{\infty e^{-i \theta}} \frac{(\log (z+z t))^{\alpha}}{t^{2}} d t=\frac{1}{\alpha} \int_{1 / z-1}^{\infty e^{-i \theta}} \frac{(\log z+\log (t+1))^{\alpha}}{t^{2}} d t
\end{aligned}
$$

Let us separate the domain of integration in two parts: $|t| \leq|\log z|$ and $|\log z| \leq|t|$. The first integral gives: 


$$
\frac{1}{\alpha} \int_{1 / z-1}^{\log z} \frac{(\log z+\log (t+1))^{\alpha}}{t^{2}} d t=\frac{(\log z)^{\alpha}}{\alpha} \int_{1 / z-1}^{\log z} \frac{(1+\log (t+1) / \log z)^{\alpha}}{t^{2}} d t
$$

Note that the numerator of the integrand satisfies:

So, the first integral gives:

$$
\left(1+\frac{\log (t+1)}{\log z}\right)^{\alpha}=1+O\left(\frac{\log \log z}{\log z}\right)=1+o(1) .
$$

$$
\frac{1}{\alpha} \int_{1 / z-1}^{\log z} \frac{(\log z+\log (t+1))^{\alpha}}{t^{2}} d t=\frac{(\log z)^{\alpha}}{\alpha}(1+o(1))
$$

For the second integral, use $(A+B)^{\alpha} \leq(2 \max \{A, B\})^{\alpha} \leq 2^{\alpha}\left(A^{\alpha}+B^{\alpha}\right)$ (valid for $A, B \geq 0$ and $\operatorname{Re}(\alpha)>0)$. It follows that we can estimate the second integral by:

$$
\begin{aligned}
\frac{1}{\alpha} \int_{\log z}^{\infty e^{-i \theta}}\left|\frac{(\log z+\log (t+1))^{\alpha}}{t^{2}}\right| d t & \leq \frac{2^{\alpha}}{\alpha} \int_{\log z}^{\infty e^{-i \theta}} \frac{|\log z|^{\alpha}}{t^{2}} d t+\frac{2^{\alpha}}{\alpha} \int_{\log z}^{\infty e^{-i \theta}} \frac{|\log (t+1)|^{\alpha}}{t^{2}} d t \\
& =O\left((\log z)^{\alpha-1}\right) .
\end{aligned}
$$

The result follows.

Remark 2.5. In fact, we can give a transseries expansion of $\operatorname{Li}_{\alpha}(z)$ for large $z$ in terms of $\log z$ and $\log \log z$.

\section{References}

[Ah] L.V. Ahlfors, Complex analysis. An introduction to the theory of analytic functions of one complex variable, Third edition, 1978.

[Ap] T. Apostol, Introduction to Analytic Number Theory, Springer-Verlag, 1976.

[BD] A. Beilinson and P. Deligne, Interprétation motivique de la conjecture de Zagier reliant polylogarithmes et régulateurs, in Motives, Proc. Sympos. Pure Math., 55, Part 2, (1994) 97-121.

[BL] A. Beilinson and A. Levin, The elliptic polylogarithm, in Motives, Proc. Sympos. Pure Math., 55 Amer. Math. Soc. (1994) 123-190.

[Bl] S. Bloch, Function theory of polylogarithms, in Structural properties of polylogarithms, Math. Surveys Monogr., 37 (1991) 275-285.

[CLZ] H. Cohen, L. Lewin and D. Zagier, A sixteenth-order polylogarithm ladder, Experiment. Math. 1 (1992) 25-34.

[Cn] J.B. Conway, Functions of one complex variable, Springer-Verlag, second edition 1978.

[C] O. Costin, Global reconstruction of analytic functions from local expansions, preprint 2007.

[CG1] and S. Garoufalidis, Resurgence of the Kontsevich-Zagier power series, preprint 2006 math.GT/0609619.

[CG2] and Resurgence of the Euler-MacLaurin summation formula, Annales de l' Institut Fourier, in press.

[CG3] and Resurgence of 1-dimensional sums of q-factorials preprint 2007.

[Df] P.A. Deift, Orthogonal polynomials and random matrices: a Riemann-Hilbert approach, Courant Lecture Notes in Mathematics, 3 New York University, 1999.

[De] P. Deligne, Valeurs de fonctions $L$ et périodes d'intégrales, Proc. Sympos. Pure Math. XXXIII (1979) 313-346.

[Ec1] J. Écalle, Resurgent functions, Vol. I Mathematical Publications of Orsay 81-05 1981, 81-06 1981 and 81-06 1981. 
[Ec2] , private communication, 09/13/2006.

[E-VG] P. Elbaz-Vincent and H. Gangl, On poly(ana)logs. I, Compositio Math. 130 (2002) 161210.

[EM] C. Epstein and J. Morava, Tempering the polylogarithm, preprint 2006 math.CA/0611240.

[Ga] S. Garoufalidis, Chern-Simons theory, analytic continuation and arithmetic, Acta Math. Vietnam. 33 (2008), no. 3, 335-362.

[Ha] R.M. Hain, Classical polylogarithms, in Motives, Proc. Sympos. Pure Math., 55, Part 2, (1994) 3-42.

[Jo] A. Jonquière, Note sur la série $\sum_{n=1}^{\infty} \frac{x^{n}}{n^{s}}$, Bull. Soc. Math. France 17 (1889) 142-152.

$[\mathrm{KW}] \quad$ R. Kenyon and D.B. Wilson, Critical resonance in the non-intersecting lattice path model, Probab. Theory Related Fields 130 (2004) 289-318.

[KZ] M. Kontsevich and D. Zagier, Periods, in Mathematics unlimited-2001 and beyond, Springer (2001) 771-808.

[Ko] , Private communication, 11/05/2006.

[Lv] A. Levin, Elliptic polylogarithms: an analytic theory, Compositio Math. 106 (1997) 267282.

[Lw] L. Lewin, Polylogarithms and associated functions, North-Holland Publishing Co. 1981.

[Ma] B. Malgrange, Introduction aux travaux de J. Écalle, Enseign. Math. 31 (1985) 261-282.

[MPV] H.N. Minh, M. Petitot and J. Van Der Hoeven, Shuffle algebra and polylogarithms, Discrete Math. 225 (2000) 217-230.

[Oe] J. Oesterlé, Polylogarithmes, Séminaire Bourbaki, Vol. 1992/93. Astérisque No. 216 (1993), Exp. No. 762 49-67.

[Ol] F. Olver, Asymptotics and special functions, Reprint. AKP Classics. A K Peters, Ltd., Wellesley, MA, 1997.

[Pi] W.F. Pickard, On polylogarithms, Publ. Math. Debrecen 15 (1968) 33-43.

[Ra] N. Ramachandran, Values of zeta functions at $s=1 / 2$, Int. Math. Res. Not. (2005), no. 25, $1519-1541$.

[Tr] C. Truesdell, On a function which occurs in the theory of the structure of polymers, Ann. of Math. 46 (1945) 144-157.

[We] G. Wechsung, Functional equations of hyperlogarithms, in Structural properties of polylogarithms, Math. Surveys Monogr., 37 AMS (1991) 171-184.

[Za1] D. Zagier, Polylogarithms, Dedekind zeta functions and the algebraic K-theory of fields, in Arithmetic algebraic geometry, Progr. Math., 89 (1991) 391-430.

[Za2] The dilogarithm function, in Frontiers in number theory, physics, and geometry. II Springer (2007) 3-65.

Department of Mathematics, Ohio State University, $231 \mathrm{~W}$ 18th Avenue, Columbus, OH 43210, USA, http://www .math.ohio-state.edu/ costin

E-mail address: costin@math.ohio-state.edu

School of Mathematics, Georgia Institute of Technology, Atlanta, Ga 30332-0160, USA, http://www.math.gatech.edu/ stavros

E-mail address: stavros@math.gatech.edu 